\title{
Closed-loop control of tumor growth by means of anti-angiogenic administration
}

\author{
Valerio Cusimano, Pasquale Palumbo, Federico Papa
}

\begin{abstract}
A tumor growth model accounting for angiogenic stimulation and inhibition is here considered, and a closed-loop control law is presented with the aim of tumor volume reduction by means of anti-angiogenic administration. To this end the output-feedback linearization theory is exploited, with the feedback designed on the basis of a state observer for nonlinear systems. The control scheme allows to set independently the control and the observer parameters thanks to the special structural properties of the tumor growth model that guarantee the separability of estimation and feedback control algorithms. Preliminary results seem extremely promising, showing a noticeable level of robustness against a wide range of the initial state estimate.
\end{abstract}

\section{INTRODUCTION}

Anti-angiogenic therapies are relatively new cancer treatments, proposed at first by Folkman [?] in the early seventies, consolidated along the nineties by several discoveries on the main principles regulating tumor angiogenesis [?] and widely debated in several theoretical and experimental studies throughout the last decade. Anti-angiogenic treatments aim at inhibiting the development of the vascular network necessary to support tumor growth during the vascular phase, so providing a way to control the heterogeneous and growthunconstrained tumor population throughout the control of the homogeneous and growth-constrained population of endothelial cells [?], [?]. Tumors have the capability to develop resistance to the conventional chemotherapeutic drugs mainly because of the rapidity of tumor cells in evolving towards new resistant phenotypes. Due to the indirect action of the anti-angiogenic drugs, the outcome of the therapy should not be impaired by the capability of tumor cells to generate resistant phenotypic variants [?], [?], and the effectiveness of anti-angiogenic treatments on the control and possible remission of experimental tumors has been demonstrated [?]. Moreover, anti-angiogenic therapies have limited side effects respect to the conventional chemotherapies and radiotherapies. Conventional chemotherapies may also have antiangiogenic effects on the vascular network [?], [?], [?].

This note aims to investigate and design a closed-loop model-based anti-angiogenic therapy. The adopted model refers to [?], where a quantitative model describing the growth of experimental tumors under the control of the vascular network is presented. In [?], Hahnfeld and coworkers introduced the concept of the carrying capacity of the

V. Cusimano, valerio.cusimanodiasi.cnr.it, P. Palumbo, pasquale.palumbodiasi.cnr.it and F. Papa federico.papaliasi.cnr.it are with Istituto di Analisi dei Sistemi ed Informatica A. Ruberti, IASI-CNR, Via dei Taurini 19, 00185 Roma, Italy vasculature, i.e. the tumor volume potentially sustainable by the vasculature, in order to account for the vascular control on the tumor growth. As the carrying capacity is strictly dependent on the vasculature extension, its dynamics can be assumed to represent the dynamics of the vascular network. Moreover, the effect on the carrying capacity of stimulatory and inhibitory angiogenic signals produced by the tumor itself and of administered anti-angiogenic drugs are explicitly accounted by the model formulation. The paper represents one of the first attempts to model, with a minimal number of parameters, the interplay between the dynamics of the tumor volume and of the carrying capacity, with or without administration of anti-angiogenic drugs. In [?] the predictions of the model have also been successfully compared with experimental data on anti-angiogenically treated and untreated Lewis lung tumors in mice.

Besides experimental frameworks, the model proposed in [?] has been widely exploited in theoretical studies in order to predict the effectiveness of new anti-angiogenic therapies and some model extensions have also been proposed in the related scientific literature. In [?] some model modifications are proposed and conditions for the eradication of the tumor under a periodic anti-angiogenic treatment are provided. In [?], [?] problems addressing the optimal scheduling of a given amount of angiogenic inhibitors are presented, while in [?] the optimal scheduling problem of a combined radiotherapy and anti-angiogenic treatment is formulated by exploiting a suitable modified version of the original model. Further extensions are also proposed in [?], where the Authors aim to describe the interplay between the populations of tumor cells and endothelial cells subject to a combined therapy of chemotherapeutic and antiangiogenic drugs. In [?] an optimal scheduling problem for the combined chemotherapeutic/anti-angiogenic treatment is presented. The model in [?] has been recently exploited to design closed-loop model-based feedback control laws in [?], [?], where robust control strategies are proposed by exploiting a Linear Quadratic controller and an $\mathrm{H}_{\infty}$ methodology applied to the linearized model.

By suitably exploiting the model in [?], the aim of tumor volume reduction is here pursued according to a closed-loop, model-based approach, with the control strategy making use of the feedback linearization theory [?]. To this end only available tumor volume measurements are exploited, with the carrying capacity estimated by means of a state observer for nonlinear system [?], [?], and the regulator is synthesized as a feedback from the observed state. The closed-loop control law is designed to track a desired low level of tumor volume, 
possibly starting from a high level situation. It is proven that the control scheme allows to set independently the control and the observer parameters, thanks to the special structural properties of the tumor growth model that guarantee the separability of estimation and feedback control algorithms. Numerical simulations revealed to be extremely promising, showing a noticeable level of robustness against a wide range of the initial state estimate and of the actual value of the carrying capacity.

The paper is organized as follows. The next section is devoted to detail the tumor growth mathematical model chosen to design the closed-loop control law, which is defined in Section 3, where the main theorem is proposed. Simulations are reported in Section 4. Conclusions follow.

\section{THE TUMOR GROWTH MODEL}

The model under investigation is a nonlinear model accounting for angiogenic stimulation and inhibition [?], and is given by the following Ordinary Differential Equations (ODE) system:

$$
\left\{\begin{array}{l}
\dot{x}_{1}=-\lambda x_{1} \ln \left(\frac{x_{1}}{x_{2}}\right) \\
\dot{x}_{2}=b x_{1}-\left(\mu+d x_{1}^{2 / 3}\right) x_{2}-c x_{2} x_{3}
\end{array}\right.
$$

where $x_{1},\left[\mathrm{~mm}^{3}\right]$, and $x_{2},\left[\mathrm{~mm}^{3}\right]$, denote the tumor volume and the carrying capacity of the vasculature, respectively. The first equation describes the phenomenology of tumor growth slowdown, as the tumor grows and resorts its available support with $\lambda,\left[\right.$ day $\left.^{-1}\right]$, denoting the tumour growth rate. In the second equation the first term represents the stimulatory capacity of the tumor upon the inducible vasculature $\left(b x_{1}\right)$, the second term accounts for spontaneous loss $\left(\mu x_{2}\right)$ and for tumor-dependent endogenous inhibition $\left(d x_{1}^{2 / 3} x_{2}\right)$ of previously generated vasculature; the third term refers to the vasculature inhibitory action performed by an exogenous drug administration $\left(c x_{2} x_{3}\right)$ with $x_{3}$, [mg/kg], denoting the serum level of the administered angiogenic inhibitor (i.e. the drug concentration). As far as the parameters, $b$, [day $\left.{ }^{-1}\right]$, is the vascular birth rate, $d,\left[\mathrm{day}^{-1} \mathrm{~mm}^{-2}\right]$, is the endothelial cell death, $\mu,\left[\right.$ day $\left.^{-1}\right]$, is the spontaneous vascular inactivation rate, and $c,\left[\mathrm{day}^{-1}(\mathrm{mg} / \mathrm{kg})^{-1}\right]$, is the sensitivity to the drug. According to the model literature [?], without loss of generality, parameter $\mu$ will be set equal to zero in the following.

Being the anti-angiogenic drug not directly administered in vein, a further compartment is considered to account for drug diffusion:

$$
x_{3}(t)=\int_{0}^{t} e^{-\eta\left(t-t^{\prime}\right)} u\left(t^{\prime}\right) d t^{\prime}
$$

with $u,\left[\right.$ day $\left.^{-1}(\mathrm{mg} / \mathrm{kg})\right]$, being the actual control law and $\eta$, $\left[\right.$ day $\left.^{-1}\right]$, being the diffusion rate into serum. As a matter of fact, the whole system (1)-(2) (with $\mu=0$ ) may be written in a compact ODE form:

$$
\left\{\begin{array}{l}
\dot{x}_{1}=-\lambda x_{1} \ln \left(\frac{x_{1}}{x_{2}}\right) \\
\dot{x}_{2}=b x_{1}-d x_{1}^{2 / 3} x_{2}-c x_{2} x_{3} \\
\dot{x}_{3}=-\eta x_{3}+u
\end{array}\right.
$$

In the following, system (3) will be also referred to as:

$$
\dot{x}=f(x)+g(x) u
$$

with $f: \mathbb{R}^{3} \mapsto \mathbb{R}^{3}$ and $g: \mathbb{R}^{3} \mapsto \mathbb{R}^{3 \times 1}$ defined by:

$$
f(x)=\left[\begin{array}{c}
-\lambda x_{1} \ln \left(\frac{x_{1}}{x_{2}}\right) \\
b x_{1}-d x_{1}^{2 / 3} x_{2}-c x_{2} x_{3} \\
-\eta x_{3}
\end{array}\right] \quad g(x)=\left[\begin{array}{l}
0 \\
0 \\
1
\end{array}\right]
$$

\section{THE CONTROL ALGORITHM}

The goal of the control law is to design a feedback control input that allows to stabilize the closed-loop system and let the tumor volume (state vaiable $x_{1}$ ) to track a desired level. The feedback is synthesized by exploiting only the tumor volume output, which is the available measurement from the model:

$$
y=h(x), \quad h: \mathbb{R}^{3} \mapsto \mathbb{R}, \quad h(x)=x_{1} .
$$

Let $r$ be the desired level of tumor volume to be tracked, smaller and safer than the non-trivial equilibrium state coming from the uncontrolled system (i.e. with $u=0$ ):

$$
x_{1, s s}=x_{2, s s}=(b / d)^{\frac{3}{2}}
$$

The control algorithm is synthesized by applying the feedback linearization theory to the nonlinear system [?], with the feedback synthesized by means of a state observer [?], [?]. To this end, consider a domain $D$ in $\mathbb{R}^{3}$ that excludes $x_{1}=0$ and $x_{2}=0$. Then, the working hypothesis concerning the state feedback linearization is satisfied, since the single-input single-output system has full relative degree in $D$. Indeed, $\forall x \in D$ it is:

$$
\begin{aligned}
& L_{g} h(x)=L_{g} L_{f} h(x)=0 \\
& L_{g} L_{f}^{2} h(x)=-\lambda c x_{1} \neq 0
\end{aligned}
$$

with $L_{f}^{k} h(x), k=1,2, \ldots$ denoting the Lie derivative of order $k$ of the scalar function $h: \mathbb{R}^{3} \mapsto \mathbb{R}$ along the vector field $f: \mathbb{R}^{3} \mapsto \mathbb{R}^{3},[?]$.

As a matter of fact [?], the following nonlinear map $\Theta$ : $\mathbb{R}^{3} \mapsto \mathbb{R}^{3}$ :

$$
\Theta(x)=\left[\begin{array}{c}
h(x)-r \\
L_{f} h(x) \\
L_{f}^{2} h(x)
\end{array}\right]=\left[\begin{array}{c}
x_{1}-r \\
-\lambda x_{1} \ln \left(x_{1} / x_{2}\right) \\
\Theta_{3}(x)
\end{array}\right]
$$

with

$$
\begin{gathered}
\Theta_{3}(x)=\lambda^{2} x_{1}\left(\ln \left(\frac{x_{1}}{x_{2}}\right)\right)^{2}+\lambda^{2} x_{1} \ln \left(\frac{x_{1}}{x_{2}}\right)+b \lambda \frac{x_{1}^{2}}{x_{2}} \\
-\lambda d x_{1}^{2 / 3} x_{1}-\lambda c x_{1} x_{3}
\end{gathered}
$$

is a diffeomorfism in $D$ with the Jacobian matrix given by

$$
J_{\Theta}=\frac{d \Theta}{d x}=\left[\begin{array}{ccc}
1 & 0 & 0 \\
-\lambda\left(1+\ln \left(x_{1} / x_{2}\right)\right) & \lambda x_{1} / x_{2} & 0 \\
J_{31}(x) & J_{32}(x) & -\lambda c x_{1}
\end{array}\right]
$$

(see the Appendix for the explicit expression of entries $J_{31}(x)$ and $\left.J_{32}(x)\right)$. Therefore, $z=\Theta(x)$ is a state transformation, and system (4) becomes, in the $z$-coordinates:

$$
\dot{z}=A_{b} z+B_{b}\left[L_{f}^{3} h\left(\Theta^{-1}(z)\right)+L_{g} L_{f}^{2} h\left(\Theta^{-1}(z)\right) u(t)\right]
$$


where $A_{b}$ and $B_{b}$ are the following Brunowski matrices

$$
A_{b}=\left[\begin{array}{lll}
0 & 1 & 0 \\
0 & 0 & 1 \\
0 & 0 & 0
\end{array}\right] \quad B_{b}=\left[\begin{array}{l}
0 \\
0 \\
1
\end{array}\right]
$$

with the explicit computation of $L_{f}^{3} h(x)$ in Appendix.

Since $L_{g} L_{f}^{2} h(x) \neq 0$, the following state feedback control law is well defined

$$
u(t)=\frac{K_{c} z(t)-L_{f}^{3} h(x(t))}{L_{g} L_{f}^{2} h(x(t))} \quad z=\Theta(x)
$$

and allows to write the closed-loop system in the $z$ coordinates as follows:

$$
\dot{z}=\left(A_{b}+B_{b} K_{c}\right) z
$$

The pair of Brunowski matrices $\left(A_{b}, B_{b}\right)$ is controllable, therefore matrix $K_{c}$ can be designed in order to make Hurwitz (i.e. eigenvalues with negative real part) the closed-loop matrix $A_{b}+B_{b} K_{c}$. This fact ensures that the $z$ components asymptotically converge to zero and, as a matter of fact, so does the first component $z_{1}=x_{1}-r$, thus ensuring the convergence of the tumor growth $x_{1}$ to the desired level $r>0$.

The drawback of such a control law is that it requires a complete knowledge of the state $x(t)$; however the carrying capacity $\left(x_{2}\right)$ and the third component $\left(x_{3}\right)$, are not directly measurable. A need exists, therefore, to exploit a state observer to overcome the problem. To this end we exploit the observer for nonlinear systems [?], [?], whose equations are:

$$
\dot{\hat{x}}=f(\hat{x})+g(\hat{x}) u(t)+J_{\Theta}(\hat{x})^{-1} K_{o}(y(t)-h(\hat{x}))
$$

where $K_{o} \in \mathbb{R}^{3 \times 1}$ is the observer gain to be designed, and $J_{\Theta}^{-1}$ is the inverse of the Jacobian matrix defined in (11), whose existence is ensured in $D$ by the full relative degree hypothesis, which implies that $\Theta$ is an observability map, and system (4) is drift-observable.

According to [?], by applying the observer equations (16) to (4) (which is drift-observable), provided that the following additional sufficient conditions hold true:

H1) $L_{f}^{3} h\left(\Theta^{-1}(z)\right)$ is uniformly Lipschitz in $\Theta(D)$, that is, there exists $\gamma_{1}$ such that $\forall z, \bar{z} \in \Theta(D)$

$$
\left\|L_{f}^{3} h\left(\Theta^{-1}(z)\right)-L_{f}^{3} h\left(\Theta^{-1}(\bar{z})\right)\right\| \leqslant \gamma_{1}\|z-\bar{z}\|
$$

H2) $L_{g} L_{f}^{2} h\left(\Theta^{-1}(z)\right)$ is uniformly Lipschitz in $\Theta(D)$, that is, there exists $\gamma_{2}$ such that $\forall z, \bar{z} \in \Theta(D)$

$$
\left\|L_{g} L_{f}^{2} h\left(\Theta^{-1}(z)\right)-L_{g} L_{f}^{2} h\left(\Theta^{-1}(\bar{z})\right)\right\| \leqslant \gamma_{2}\|z-\bar{z}\|
$$

H3) the input $u$ is uniformly bounded, that is:

$$
\sup _{t \geq 0}\|u(t)\|<M<+\infty
$$

then, there exists an observer matrix $K_{o} \in \mathbb{R}^{3 \times 1}$, such that the state estimate converges asymptotically to the real state if the initial error is sufficiently small.
Differently than (14), by straightforwardly applying the state observer (16) to design the closed loop control, the control input would be given by:

$$
\hat{u}(t)=\frac{K_{c} \hat{z}(t)-L_{f}^{3} h(\hat{x}(t))}{L_{g} L_{f}^{2} h(\hat{x}(t))} \quad \hat{z}=\Theta(\hat{x})
$$

Though implementable by means of only measured state variables, such a control law would not ensure the separability principle, since it is well known that the separability principle does not hold true for nonlinear systems, unless special cases. The following Theorem shows how to modify the control scheme in order to elude such a drawback.

Theorem 1. Consider the control input:

$$
u(t)=\frac{K_{c} \hat{z}(t)-L_{f}^{3} h(\hat{x}(t))}{H(y(t))}, \quad \hat{z}=\Theta(\hat{x})
$$

with $H(y)=-\lambda c y$ and $\hat{x}(t)$ provided by:

$\dot{\hat{x}}=f(\hat{x})+J_{\Theta}(\hat{x})^{-1} B_{b} H(y) u(t)+J_{\Theta}(\hat{x})^{-1} K_{o}(y(t)-h(\hat{x}))$

Then, provided that hypothesis $\mathrm{H} 1$ ) holds true, it is possible to design the gain matrix $K_{c}$ in (21) and the observer gain $K_{o}$ in (22) in order to ensure the asymptotic convergence of the observer (by means of $K_{o}$ ) independently of the expontial convergence of $x_{1}(t) \mapsto r$ (by means of $K_{c}$ ).

Proof: The proof is organized in two steps. Step 1 shows that it is possible to design the observer gain in order to ensure the asymptotic convergence to zero of the observer error; Step 2 shows how to design the control gain $K_{c}$ independently of $K_{o}$ in order to ensure the exponential convergence of the tumor growth $x_{1}$ to the desired level $r$.

Step 1. Define $C_{b}=\left[\begin{array}{lll}1 & 0 & 0\end{array}\right]$. By exploiting the definition of $\hat{z}$ in (21), the observer equation (22) can be written in the $\hat{z}$-coordinates as:

$$
\begin{aligned}
\dot{\hat{z}}= & {\left[\frac{d \Theta}{d x} \dot{\hat{x}}(t)\right]_{\hat{x}=\Theta^{-1}(\hat{z})}=\left[J_{\Theta}(\hat{x}) \dot{\hat{x}}(t)\right]_{\hat{x}=\Theta^{-1}(\hat{z})} } \\
=A_{b} \hat{z}+B_{b} L_{f}^{3} h\left(\Theta^{-1}(\hat{z})\right)+B_{b} H(y) u(t) & +K_{o}\left(y(t)-C_{b} \hat{z}\right)
\end{aligned}
$$

where the identity

$$
\left.\left[J_{\Theta}(x) f(x)\right]\right|_{x=\Theta^{-1}(z)}=A_{b} z+B_{b} L_{f}^{3} h\left(\Theta^{-1}(z)\right)
$$

has been properly used.

Notice that the nonlinear function $H(y)$ coincides with $L_{g} L_{f}^{2} h(x)$, since this Lie derivative only depends on the first (measured) coordinate $x_{1}$, see (8). As a matter of fact, the $z$-coordinate system (12) can be rewritten as:

$$
\dot{z}=A_{b} z+B_{b} L_{f}^{3} h\left(\Theta^{-1}(z)\right)+B_{b} H(y) u(t)
$$

Hence, we readily obtain that the observer error in the $z$ coordinates $e_{z}(t)=z(t)-\hat{z}(t)$ does not depend on the control input $u$ :

$\dot{e}_{z}=\left(A_{b}-K_{o} C_{b}\right) e_{z}+B_{b}\left[L_{f}^{3} h\left(\Theta^{-1}(z)\right)-L_{f}^{3} h\left(\Theta^{-1}(\hat{z})\right)\right]$ 
By exploiting (26), it has been proven in [?] that:

(i) according to the sufficient hypothesis $\mathrm{H} 1$ ), the $e_{z}$ dynamics obeys the following inequality:

$$
\begin{array}{r}
\left\|e_{z}(t)\right\| \leq e^{\left(\sigma_{1}+\sqrt{3} \gamma_{1}\left\|V^{-1}(\sigma)\right\|\right) t}\left\|V^{-1}(\sigma)\right\| \\
\cdot\|V(\sigma)\| \cdot\left\|e_{z}(0)\right\|
\end{array}
$$

where $\sigma=\left\{\sigma_{1}, \sigma_{2}, \sigma_{3}\right\}$ are distinct real eigenvalues properly assigned by $K_{o}$ to matrix $A_{b}-K_{o} C_{b}$, with $\sigma_{1}$ the greater eigenvalue and

$$
V(\sigma)=\left[\begin{array}{lll}
\sigma_{1}^{2} & \sigma_{1} & 1 \\
\sigma_{2}^{2} & \sigma_{2} & 1 \\
\sigma_{3}^{2} & \sigma_{3} & 1
\end{array}\right]
$$

is the Vandermonde matrix associate to spectrum $\sigma$;

(ii) for any chosen $\alpha>0$, there exist a way to choose negative real eigenvalues $\sigma_{3}<\sigma_{2}<\sigma_{1}<0$ such that:

$$
\sigma_{1}+\sqrt{3} \gamma_{1}\|V(\sigma)\|<-\alpha
$$

These facts ensure that the observer gain $K_{o}$ can be designed in order to have exponential convergence to zero of the $e_{z}$ error, (27), and, therefore, asymptotic convergence to zero of the error $e(t)=x(t)-\hat{x}(t)$, according to the continuity of $\Theta^{-1}$.

Step 2. By substituting the control input (21) in (25), we obtain:

$$
\begin{aligned}
\dot{z}=\left(A_{b}+B_{b} K_{c}\right) z-B_{b} K_{c} e_{z} & \\
+ & B_{b}\left[L_{f}^{3} h\left(\Theta^{-1}(z)\right)-L_{f}^{3} h\left(\Theta^{-1}(\hat{z})\right)\right]
\end{aligned}
$$

According to (30), we can write the following integral equation

$$
\begin{aligned}
z(t) & =e^{\left(A_{b}+B_{b} K_{c}\right) t} z(0)-\int_{0}^{t} e^{\left(A_{b}+B_{b} K_{c}\right)(t-\tau)} B_{b} K_{c} e_{z}(\tau) d \tau \\
& +\int_{0}^{t} e^{\left(A_{b}+B_{b} K_{c}\right)(t-\tau)} B_{b}[\alpha(z(\tau))-\alpha(\hat{z}(\tau))] d \tau
\end{aligned}
$$

where $\alpha(\cdot)=L_{f}^{3} h\left(\Theta^{-1}(\cdot)\right)$. Now, let $\nu=U(\xi) z$ with $U(\xi)$ the Vandermonde matrix associated to the spectrum $\xi=\left\{\xi_{1}, \xi_{2}, \xi_{3}\right\}$ of distinct real eigenvalues assigned by the gain matrix $K_{c}$ to $A_{b}+K_{c} B_{b}$. Then, by denoting

$$
\Xi=\operatorname{diag}\left\{\xi_{1}, \xi_{2}, \xi_{3}\right\}=U(\xi)\left(A_{b}+B_{b} K_{c}\right) U^{-1}(\xi)
$$

we have:

$$
\begin{aligned}
\nu(t) & =e^{\Xi t} \nu(0)-\int_{0}^{t} e^{\Xi(t-\tau)} U(\xi) B_{b} K_{c} e_{z}(\tau) d \tau \\
& +\int_{0}^{t} e^{\Xi(t-\tau)} U(\xi) B_{b}[\alpha(z(\tau))-\alpha(\hat{z}(\tau))] d \tau
\end{aligned}
$$

and, according to hypothesis H1):

$$
\begin{aligned}
\|\nu(t)\| \leqslant e^{\xi_{1} t} & (\|\nu(0)\| \\
& \left.+\int_{0}^{t} e^{-\xi_{1} \tau} \sqrt{3}\left(\gamma_{1}+\left\|K_{c}\right\|\right)\left\|e_{z}(\tau)\right\| d \tau\right)
\end{aligned}
$$

where $\xi_{1}$ is the greatest eigenvalue in $\xi$ and the identity $\left\|U(\xi) B_{b}\right\|=\sqrt{3}$ (straightforwardly coming from the definitions of the Vandermonde matrix, (28), and $B_{b}$, (13)) has been properly considered. Finally, by exploiting inequality (27), and according to a proper choice for $K_{o}$ in order to ensure inequality (29), (34) becomes

$$
\|\nu(t)\| \leqslant e^{\xi_{1} t}\left(\|\nu(0)\|+\int_{0}^{t} \Omega e^{-\left(\xi_{1}+\alpha\right) \tau} d \tau\right)
$$

with

$$
\Omega=\sqrt{3}\left(\gamma_{1}+\left\|K_{c}\right\|\right) \cdot\left\|V^{-1}(\sigma)\right\| \cdot\|V(\sigma)\| \cdot\left\|e_{z}(0)\right\|
$$

After the computation of the integral:

$$
\|\nu(t)\| \leqslant e^{\xi_{1} t}\|\nu(0)\|+\frac{\Omega}{\alpha+\xi_{1}}\left(e^{\xi_{1} t}-e^{-\alpha t}\right)
$$

Therefore, by properly designing $K_{c}$, independently from $K_{o}$, in order to have $\xi_{1}<0$, it is $\|\nu(t)\| \mapsto 0$. Thus $z(t)$ converges to zero exponentially since:

$$
\|z(t)\| \leqslant\left\|U^{-1}(\xi)\right\| \cdot\|\nu(t)\|
$$

and this implies that $x_{1}(t) \mapsto r$ exponentially as well, since $x_{1}-r$ is the first component of $z$.

Remark 2. Notice that, in case $\Theta^{-1}$ is Lipschitz in $\Theta(D)$, that is

$$
\left\|\Theta^{-1}(z)-\Theta^{-1}(\bar{z})\right\| \leqslant \gamma_{3}\|z-\bar{z}\| \quad \forall z, \bar{z} \in \Theta(D)
$$

then the observer error $e(t)=x(t)-\hat{x}(t)$ converges to zero exponentially, since

$$
\|e(t)\| \leq \gamma_{3}\left\|e_{z}(t)\right\| \mapsto 0
$$

\section{Simulation RESUTS}

To demonstrate the validity of the algorithm, simulations are performed setting the model parameters to the values estimated in [?]. Such an estimation was based on experimental data of Lewis lung carcinoma implanted in C57BL/6 mice. The data referred to volume measurements of both untreated control tumors and treated tumors under a regimen with endostatin. Treatment was initiated when tumors were $\sim 200 \mathrm{~mm}^{3}$ in size and it ended after 13 days. The treatment regimen was $20 \mathrm{mg} / \mathrm{kg} / \mathrm{day}$. The produced fitting curve showed a final tumor volume of $\sim 135 \mathrm{~mm}^{3}$, setting the initial value of the carrying capacity to $625 \mathrm{~mm}^{3}$. The parameters estimated in [?], are reported in Table IV.

TABLE I

MOdel PARAMETERS
\begin{tabular}{|c|c|c|c|c|}
\hline $\begin{array}{c}\lambda \\
\text { day }^{-1}\end{array}$ & $\begin{array}{c}b \\
\mathrm{day}^{-1}\end{array}$ & $\begin{array}{c}d \\
\mathrm{day}^{-1} \mathrm{~mm}^{-2}\end{array}$ & $\begin{array}{c}\mu \\
\mathrm{day}^{-1}\end{array}$ & $\begin{array}{c}c \\
\mathrm{day}^{-1}(\mathrm{mg} / \mathrm{kg})^{-1}\end{array}$ \\
\hline 0.192 & 5.85 & 0.00873 & 0 & 0.66 \\
\hline
\end{tabular}

According to [?], the treatment length has been fixed to 13 days, and the desired tumor level $r$ has been set equal to $135 \mathrm{~mm}^{3}$ with the goal to get close to $r$ at the end of the treatment.

Simulations have been carried out by setting the initial tumor volume $x_{1}(0)$ equal to $200 \mathrm{~mm}^{3}$ and by varying the initial value of the carrying capacity $x_{2}(0)$ in the percentage 
range of $[-40,+200] \%$ with respect to the reference value of $625 \mathrm{~mm}^{3}$. The initial value of the serum level of the administered angiogenic inhibitor $x_{3}(0)$ is set equal to zero. Also these initial values are consistent with real data coming from [?].

For the observer initialization, $\hat{x}_{1}(0)$ is set equal to the measurement of the tumor volume at day zero, $\hat{x}_{3}(0)$ is set equal to zero, and $\hat{x}_{2}(0)$ is allowed to vary in the percentage range of $[-40,+200] \%$ with respect to real value $x_{2}(0)$.

For each pair of initial conditions $\left(x_{2}(0), \hat{x}_{2}(0)\right)$, three different targets have been address that is:

1) to keep the final value of the tumor volume within $10 \%$ of variation of the reference level:

$$
x_{1}(13 \Delta) \in[0.9 r, 1.1 r], \quad \Delta=1 \text { day }
$$

2) to keep the total amount of the administered drug smaller than $260 \mathrm{mg} / \mathrm{kg}$ (i.e. $20 \mathrm{mg} / \mathrm{kg}$ per 13 day):

$$
m=\int_{0}^{13 \Delta} u(t) d t \leqslant 260
$$

3) to keep the average daily amount of the administered drug within $40 \%$ of variation with respect to 20 $\mathrm{mg} / \mathrm{kg} /$ day:

$$
m_{d}=\frac{1}{\Delta} \int_{T}^{(T+1) \Delta} u(t) d t=20 \cdot(1 \pm 40 \%)
$$

with $T=0, \ldots, 12$,

Matrices $K_{c}$ and $K_{o}$ are designed in order to ensure the eigenvalues of $A_{b}-K_{o} C_{b}$ and $A_{b}-B_{b} K_{c}$ to be equal to $[-0.45,-0.9,-1.35]$ and $[-1,-2,-3]$, respectively. The same set of control parameters has been considered for all simulations reported.

Accounting for different initial conditions of $x_{2}$ and $\hat{x}_{2}$, Table II and Table III report the values of $x_{1}(13 \Delta)$ and $m$, respectively, and Table IV refers to the maximum value (out of 13 average daily delivered amounts) of $m_{d}$.

TABLE II

\begin{tabular}{|c|c|c|c|c|c|c|c|}
\hline & \multicolumn{6}{|c|}{$\Delta x_{2}(0)(\%)$} \\
\hline & & -40 & -20 & 0 & 50 & 100 & 200 \\
\hline \multirow{6}{*}{$0^{\circ}$} & -40 & 139.13 & 139.19 & 139.25 & 139.35 & 139.36 & 139.39 \\
\hline & -20 & 139.09 & 138.97 & 138.94 & 138.62 & 138.46 & 138.80 \\
\hline & 0 & 138.73 & 138.62 & 138.34 & 137.82 & 137.86 & 138.42 \\
\hline & 50 & 137.76 & 137.11 & 136.88 & 136.95 & 137.23 & 137.96 \\
\hline & 100 & 136.45 & 136.17 & 136.15 & 136.46 & 136.87 & 137.66 \\
\hline & 200 & 135.15 & 135.21 & 135.39 & 135.91 & 136.40 & 137.25 \\
\hline
\end{tabular}

FinAL VALUE OF TUMOR VOLUME $x_{1}(13 \Delta)$, AT THE END OF THE TREATMENT

The tables demonstrate the good performance of the algorithm, in spite of the great range of variability of the carrying capacity and of its estimate. Looking at Table II it may be appreciated that the final value of the tumor volume is very close to the desired value of $135 \mathrm{~mm}^{3}$, with its variability lower than $4 \%$. Table III ensures a total amount smaller than the upper bound of $260 \mathrm{~mm}^{3}$, compatible with the total amount delivered in the experiments of [?]. As far as Table IV, even if it tells of an average daily delivery

\begin{tabular}{|c|c|c|c|c|c|c|c|}
\hline & \multicolumn{6}{|c|}{$\Delta x_{2}(0)(\%)$} \\
\hline & & -40 & -20 & 0 & 50 & 100 & 200 \\
\hline \multirow{6}{*}{ 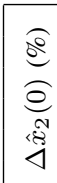 } & -40 & 247.78 & 249.34 & 250.90 & 253.89 & 256.10 & 259.35 \\
\hline & -20 & 247.39 & 248.82 & 249.73 & 251.68 & 253.37 & 257.53 \\
\hline & 0 & 247.00 & 247.65 & 248.04 & 249.47 & 251.81 & 256.36 \\
\hline & 50 & 242.97 & 242.71 & 243.62 & 246.61 & 249.60 & 254.67 \\
\hline & 100 & 238.94 & 240.11 & 241.54 & 245.18 & 248.43 & 253.76 \\
\hline & 200 & 235.95 & 237.90 & 239.85 & 243.88 & 247.26 & 252.59 \\
\hline
\end{tabular}

TABLE III

TOTAL AMOUNT $m$ OF THE ADMINISTERED ANTI-ANGIOGENIC DRUG

\begin{tabular}{|c|c|c|c|c|c|c|c|}
\hline & \multicolumn{6}{|c|}{$\Delta x_{2}(0)(\%)$} \\
\hline & & -40 & -20 & 0 & 50 & 100 & 200 \\
\hline \multirow{6}{*}{$\underset{\substack{\mathbb{d} \\
\text { ed }}}{\stackrel{0}{e}}$} & -40 & 26.24 & 26.44 & 26.61 & 26.95 & 27.16 & 27.50 \\
\hline & -20 & 26.14 & 26.30 & 26.36 & 26.47 & 26.59 & 27.13 \\
\hline & 0 & 26.03 & 26.02 & 25.98 & 25.96 & 26.21 & 26.91 \\
\hline & 50 & 25.16 & 24.85 & 24.83 & 25.17 & 25.65 & 26.51 \\
\hline & 100 & 24.10 & 24.06 & 24.20 & 24.77 & 25.34 & 26.27 \\
\hline & 200 & 22.98 & 23.24 & 23.56 & 24.33 & 24.98 & 25.96 \\
\hline
\end{tabular}

TABLE IV

MAXIMUM AVERAGE DAILY AMOUNT $m_{d}$ OF ANTI-ANGIOGENIC DRUG

greater than $20 \mathrm{~mm}^{3}$, such delivery never shows a variability greater than $40 \%$. A further comment is that best results in terms of Table II and Table IV are obtained when $\hat{x}_{2}(0)$ is overestimated. As expected the performance of the controller worsen when $x_{2}(0)$ is high.

Finally, all tables deal with a control algorithm that shows a noticeable level of robustness with respect to the initial condition of the carrying capacity, as well as to its estimate.

In Fig. 1 an example of the system dynamics is presented.

\section{CONCLUSIONS}

Based on a mathematical model of tumor growth, this work proposes a closed-loop control law aiming to reduce the tumor volume. The model accounts for angiogenic stimulation and inhibition, and is one of the most adopted in the literature to simulate and predict the effects of antiangiogenic drug delivery. Based on the feedback linearization theory, the control law makes use of an observer for nonlinear systems in order to design the model-based control by means of only available measurements. Theoretical results ensure that the control gain of the regulator can be set independently of the observer gain, thanks to the structural properties of the tumor growth model. Numerical simulations show the effectiveness of the control law in spite of a wide range of variation of the (not measured) carrying capacity.

\section{APPENDIX}

In this section the explicit expression of entries $J_{31}(x)$, $J_{32}(x)$ in (11) and $L_{f}^{3} h(x)$ are reported.

$$
\begin{aligned}
J_{31}(x)= & \lambda^{2}\left(3 \ln \left(\frac{x_{1}}{x_{2}}\right)+\ln \left(\frac{x_{1}}{x_{2}}\right)^{2}\right) \\
& -\frac{5}{3} \lambda d x_{1}^{\left(\frac{2}{3}\right)}+\lambda^{2}-\lambda c x_{3}+\frac{2 \lambda b x_{1}}{x_{2}} ; \\
J_{32}(x)=- & \frac{\lambda^{2} x_{1}}{x_{2}}\left(2 \ln \left(\frac{x_{1}}{x_{2}}\right)+1\right)-\frac{\lambda b x_{1}^{2}}{x_{2}^{2}} ;
\end{aligned}
$$



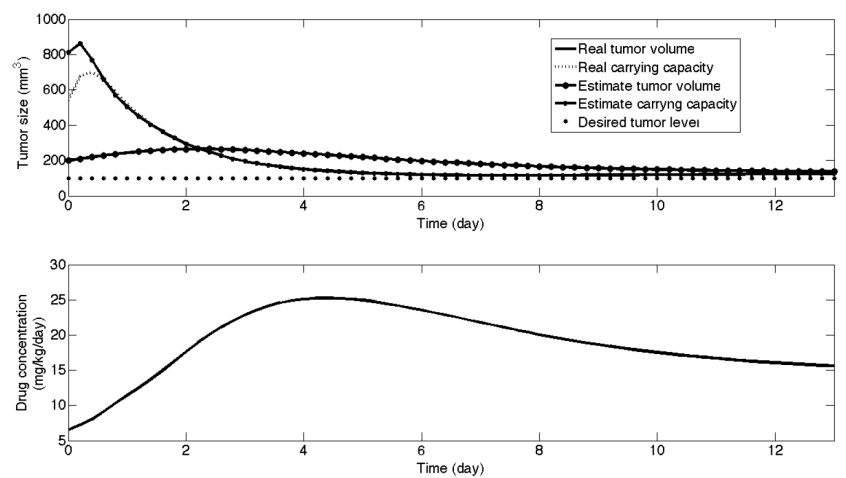

Fig. 1. Graphical comparison of the real and estimate state under the action of the closed loop control law

$$
\begin{gathered}
L_{f}^{3} h(x)=\phi(x) \lambda \frac{x_{1}}{x_{2}}\left(2 \lambda \ln \left(\frac{x_{1}}{x_{2}}\right)-\phi(x)+c x_{3}+d x_{1}^{\frac{2}{3}}+\lambda\right) \\
-\lambda^{2} \frac{x_{1}^{2}}{x_{2}}\left[\lambda \frac{x_{1}}{x_{2}}+\lambda\left(1+2 \ln \left(\frac{x_{1}}{x_{2}}\right)+\left(\ln \left(\frac{x_{1}}{x_{2}}\right)\right)^{2}\right)\right. \\
\left.-\frac{5}{3} d x_{1}^{\frac{2}{3}}+2 b \frac{x_{1}}{x_{2}}-c x_{3}\right]-\lambda c \eta x_{1} x_{3}
\end{gathered}
$$

with

$$
\phi(x)=d x_{1}^{\frac{2}{3}} x_{2}-b x_{1}+c x_{2} x_{3}
$$

\section{REFERENCES}

